\title{
MAPEAMENTO SISTEMÁTICO SOBRE UMA ANÁLISE DO IMPACTO DAS PRÁTICAS DE BIG DATA NA ADMINISTRAÇÃO PÚBLICA FEDERAL
}

\author{
Fernando Gonçalves de Oliveira ${ }^{1}$, Washington Henrique Carvalho Almeida ${ }^{1}$, \\ Luciano de Aguiar Monteiro ${ }^{1}$, Fernando Escobar ${ }^{2}$, \\ Rodrigo Otávio Ribeiro Hagstrom ${ }^{2}$ e Daniel Alves da Silva ${ }^{2}$ \\ ${ }^{1}$ CESAR School, Cais do Apolo, 77, Bairro Recife - Recife-PE, CEP:50030-390, Brasil \\ ${ }^{2}$ Universidade de Brasília - UNB, Campus Universitário Darcy Ribeiro, Brasília -DF, CEP: 70910-900, Brasil
}

\begin{abstract}
RESUMO
Este artigo apresenta um mapeamento sistemático da literatura referente à análise do impacto das práticas de Big Data na Administração Pública Federal, com a premissa de que as instituições públicas guardam semelhanças em missões institucionais, ainda que em locais diferentes e em setores diferentes. O principal objetivo dessa pesquisa é reunir subsídios para responder quais os objetivos e os maiores desafios acerca da implantação de Big Data e/ou Ecossistemas de Big Data, nos órgãos públicos federais brasileiros. O levantamento dos artigos foi realizado em três bases de dados (ACM Digital Library, IEEE Xplore e Science Direct - Elsevier), utilizando strings de busca, a fim filtrar um conjunto de artigos que estejam alinhados com os tópicos e respostas para as questões de pesquisa. Este mapeamento sistemático é uma etapa balizadora de uma pesquisa mais ampla que, em suma, pode direcionar estruturações de projetos de Big Data no setor público, em especial na Administração Pública Federal brasileira. Compreendeu-se que a implantação de Big Data no setor público precisa estar perfeitamente alinhada com a missão institucional do órgão que se propõe a implantá-lo, mas que os gestores públicos nem sempre percebem suas instituições como aptas a implantarem projetos desta natureza. Tal problema pode demandar que seja apresentado todo um conjunto de diferentes projetos de Big Data em diferentes esferas e missões institucionais. Finalmente, se percebe que há pouca literatura relacionada com o caso brasileiro, o que evidencia a pertinência, tanto do mapeamento sistemático quanto de etapas futuras da pesquisa.
\end{abstract}

\section{PALAVRAS-CHAVE}

Big Data, Administração Pública Federal, Ecossistema de Big Data, Mapeamento Sistemático

\section{INTRODUÇÃO}

Atualmente, indivíduos e organizações, sejam privadas, públicas ou do terceiro setor, vêm gerando grandes volumes de dados, em uma proporção que cresce constantemente (Akoka et al., 2017). Complementarmente, a popularização dos dispositivos conectados (Internet of Things - IoT), sensores e aplicações que coletam e geram dados em forma de imagem, áudio e vídeo, tem contribuído significativamente para esse volume e sua curva ascendente. Esse contexto ganha impulso, relevância e complexidade com o advento da quarta revolução industrial, conhecida como Indústria 4.0 (Akoka et al., 2017; Almeida et al., 2019).

Big Data é um termo genérico para a enorme quantidade de dados digitais não estruturados, normalmente coletados de múltiplas fontes; o termo também representa os novos desafios que envolvem a complexidade, a segurança e os riscos à privacidade, bem como a necessidade de novas tecnologias e habilidades humanas, redefinindo o cenário do gerenciamento de dados e dos processos de extração, transformação e carregamento (Kim et. al., 2014).

Akoka et al. (2017) realizaram um trabalho de enfoque meta analítico com artigos sobre Big Data, o qual demonstra que a quantidade de estudos sobre o termo vem crescendo, o que pode estar relacionado com o aumento da percepção acerca da importância de tal conjunto de tecnologias e de sua implantação. Para responder à questão sobre quais os assuntos produzidos a partir das pesquisas de Big Data, os autores classificaram os trabalhos pesquisados em domínios de conhecimento, abrangendo, entre outros, energia, medicina, ecologia, finanças e governo. A presença do domínio "governo" demonstra a relevância do tema em 
relação a pesquisas com Big Data. Entretanto, de um total de 441 trabalhos analisados, apenas 35 pesquisas reportaram casos governamentais, posicionando esse tópico na oitava posição de um universo de doze domínios. Isso evidencia a carência de trabalhos relacionados ao domínio "governo" ou "setor público" e uma série de oportunidades que podem ser exploradas.

No caso do Brasil, há evolução no entendimento de que os órgãos do governo federal devem estar envolvidos em um ambiente que permita a adequada transparência das atividades públicas, bem como a necessidade de controle e conhecimento de informações, em larga escala, para melhor gestão das atividades relacionadas com os serviços públicos e governamentais (Victorino et al., 2017). Os autores complementaram seu trabalho apresentando uma proposta de Ecossistema de Big Data, para dar suporte a análise de dados abertos governamentais, como resposta ao contexto de transparência. Estas características sugerem que o caso brasileiro guarda semelhanças com os casos internacionais mais ricamente presentes na literatura correlata, bem como semelhanças entre os órgãos brasileiros de diferentes setores, o que se tornou uma premissa no trabalho relatado neste artigo.

Este artigo tem como tema o mapeamento sistemático referente à análise do impacto das práticas de Big Data na Administração Pública Federal do Brasil, ocorrido ou a ocorrer, abordando o tema por meio de pensamentos de autores que delineiam as bases para o estabelecimento do ecossistema de Big Data no setor público e as boas práticas relacionadas. Assim, o objetivo principal é entender quais os propósitos e os maiores desafios e dificuldades relacionados com a adoção do Big Data na Administração Pública (AP). A pesquisa permite identificar inciativas e a visão dos ecossistemas de Big Data que possam auxiliar ambientes profissionais ou organizacionais com aplicabilidade prática. O estudo em tela atinge, inicialmente, esse objetivo, sendo o ambiente profissional, no caso, a Administração Pública.

O restante deste artigo está estruturado da seguinte forma: a Seção 2 aborda alguns conceitos centrais para o entendimento da terminologia que compõe o cenário de Big Data e a Seção 3 apresenta a Metodologia da pesquisa. $\mathrm{Na}$ Seção 4, são apresentadas as conclusões deste mapeamento sistemático.

\section{CONCEITOS}

Segundo Victorino et al. (2017), citando Schimdt (2010), a humanidade criou, até o ano de 2003, cerca de cinco exabytes de dados. O volume, desde então, dobra a cada dois anos em média (Gantz and Reinsel, 2011), o que fez com que no ano de 2019 fossem criados cerca de 160 exabytes de dados. Consolidando o conceito de Big Data, nas palavras de Araújo Júnior and Souza (2018), fundamentados em Ramos (2015), Big Data é um conjunto de tecnologias utilizadas para tratar grandes volumes de dados. É importante destacar que, não necessariamente, os dados tomados para tratamento estão em formatos similares, compatíveis. Em um cenário como este, conforme destacam os mesmos autores, tornam-se necessárias soluções computacionais mais efetivas em termos de custos e mais inovadoras no tratamento da informação.

Victorino et al. (2017) ainda destacam, como uma destas soluções computacionais, o Big Data, o qual possibilita a gestão de dados em grande velocidade de processamento, mas que não se refere especificamente à sua organização. Araújo Júnior and Souza (2018) destacam que o conceito de Big Data tem sua origem no Business Intelligence (BI), que é, por sua vez, resultado do processo de mineração de dados analíticos, voltado para a combinação de dados, formatação de metadados e de conteúdo a serem usados no processo decisório. O BI é o uso de variadas fontes de dados e informações, utilizadas para definir estratégias e subsidiar processos organizacionais. Logo, os conceitos de Big Data e BI convergem e, por isso, alguns autores consideram as duas concepções como apenas uma (Araújo Júnior and Souza, 2018; Barbieri, 2001).

Araújo Júnior and Souza (2018), Davenport (2014), Márquez and Lev (2017) são outros que também consideram os conceitos similares. Entretanto, não se verifica que os autores se preocupem em citar a organização dos dados utilizados nos processos, nem mesmo acerca do armazenamento. Ainda, conforme Inmon and Linstedt (2001), os conceitos são similares quando em relação a modelos que envolvem a captura, apresentação e geração de relatórios para a tomada de decisão.

Davenport (2014), citado por Victorino et al. (2017), afirma que o termo Big Data é muito genérico, utilizado para conjuntos de dados que não são comportados em repositórios habituais. Logo, o termo estaria relacionado a conjuntos de dados massivos e volumosos. Não caberiam, portanto, em servidores tradicionais e bancos de dados relacionais, demandando novas tecnologias de armazenagem e tratamento. 
É comum, nos artigos analisados, que os autores abordem a definição de "3Vs" (Akoka et al., 2017; Araújo Júnior and Souza, 2018; Davenport, 2014; Laney, 2001), caracterizando o Big Data, quaisquer que sejam, conforme definições presentes em Araújo Júnior and Souza (2018):

- volume: permite a modelagem e a análise de extensos volumes de dados e o que será extraído e armazenado;

- velocidade: fornece resultados de pesquisa com agilidade, em tempo compatível com a demanda, na qual os dados serão gerados, a rapidez na ação tomada sob os dados ou a taxa de mudança nos dados;

- variedade: processa e fornece conjuntos de dados em diferentes tipos e formatos.

A estes "3Vs", Davenport (2014) acrescentou, posteriormente, como destacam Victorino et al. (2017), outros dois "Vs", totalizando, então, os "5Vs" da definição do Big Data. São os outros dois "Vs", também na definição de Araújo Júnior and Souza (Araújo Júnior and Souza, 2018; Hashem et al., 2015):

- veracidade: processa e fornece conjuntos de dados fiéis aos dados processados e armazenados nos bancos, diminuindo incertezas e aumentando a confiabilidade dos dados; e

- valor: agrega valor informacional; informação de valor claro para as organizações que as utilizam.

A compilação dos “5Vs" é graficamente representada na Figura 1.

\begin{tabular}{|c|c|c|c|c|}
\hline Volume & Variedade & Velocidade & Veracidade & Valor \\
\hline $\begin{array}{c}\text { Q貝 } \\
\text { 三uantidade de } \\
\text { dados que são } \\
\text { gerados a cada } \\
\text { segundo }\end{array}$ & $\begin{array}{c}\text { (1) } \\
\text { ques de dados } \\
\text { utilizados }\end{array}$ & $\begin{array}{c}\text { Velocidade a qual } \\
\text { os dados são } \\
\text { gerados e } \\
\text { transmitidos }\end{array}$ & $\begin{array}{c}\text { 吅品 } \\
\text { 四品 } \\
\text { Grau de } \\
\text { organização e } \\
\begin{array}{c}\text { confiabilidade dos } \\
\text { dados }\end{array}\end{array}$ & $\begin{array}{l}\text { Necessidade de } \\
\text { se transformar } \\
\text { dados em valor }\end{array}$ \\
\hline $\begin{array}{l}\text { Terabytes } \\
\text { Registros } \\
\text { Transações } \\
\text { Arquivos }\end{array}$ & $\begin{array}{c}\text { Estruturado } \\
\text { Semiestruturado } \\
\text { Não estruturado }\end{array}$ & $\begin{array}{l}\text { Tempo real } \\
\text { Streams } \\
\text { Batch }\end{array}$ & $\begin{array}{l}\text { Fidedignidade } \\
\text { Autenticidade } \\
\text { Origem } \\
\text { Relevância } \\
\text { Disponibilidade }\end{array}$ & $\begin{array}{l}\text { Estatística } \\
\text { Eventos } \\
\text { Correlações } \\
\text { Hipotética }\end{array}$ \\
\hline
\end{tabular}

Figura 1. Demonstração de Big Data por meio dos 5Vs

O processamento de grandes massas de dados é um dos principais motivos pelos quais gestores têm buscado projetos de Big Data (Almeida et al., 2019). Portanto, o provimento de capacidade analítica a partir da extração de novas visões derivadas de grandes volumes de dados, o que em bases de dados tradicionais pode ser considerado complexo, bem como as demais estruturas que compõem o Ecossistema de Big Data.

\subsection{Ecossistema de Big Data}

Segundo Victorino et al. (2017), revisitando os conceitos elencados em Shin and Choi (2015), assim como em outras ciências, o conceito de ecossistema estaria relacionado com as interações entre os diversos componentes de um sistema. No contexto do Big Data, portanto, estaria relacionado com as interações entre tecnologia, governo, indústria, mercados, usuários e sociedade, sendo examinados ou investigados os efeitos do ambiente de Big Data em todos os setores envolvidos. Victorino et al. (2017) citam, ainda, a similaridade desta definição com a encontrada em Demchenko et al. (2014), na qual a abordagem biológica é mantida, mas com a diferença na definição do ecossistema, como um complexo de facilidades técnicas e componentes construídos acerca de certa origem de dados específica e de suas aplicações, sendo que o complexo de componentes inter-relacionados é voltado para as atividades de armazenamento, processamento, visualização e entrega dos resultados. O ecossistema compreenderia, ainda, alguns componentes arquiteturais, tecnologias e outros componentes para o processamento e a análise do Big Data, devendo compreender o gerenciamento do seu ciclo de vida, ou seja, o fluxo de transformação dos dados e toda sua infraestrutura de segurança, bem como 
todo o conjunto necessário de componentes e políticas para prover controle no acesso aos dados (Almeida et al., 2019).

É possível observar, se tomadas as explicações em epígrafe, que os dados não se encontram em repositórios tradicionais, que são oriundos de diversas fontes, que o volume de dados é massivo (Almeida et al., 2019) e que Big Data e Cloud Computing (ou Computação em Nuvem, em língua portuguesa) estão relacionados. Esta relação é parte do trabalho de Hashem et al. (2015), no qual os autores detalham que Big Data e Cloud Computing se complementam, fornecendo aos usuários a capacidade de utilizar poder computacional em larga escala para processar consultas distribuídas em vários conjuntos de dados e devolver as resultantes em tempo hábil. De forma subjacente, a Computação em Nuvem fornece outros mecanismos como, por exemplo, o do framework Hadoop e do MapReduce.

O MapReduce (Dean and Ghemawat, 2008) é um exemplo de aplicação de Big Data em um ambiente de Computação em Nuvem, o qual permite o processamento de grandes quantidades de conjuntos de dados, armazenados em paralelo no cluster Hadoop (Almeida et al., 2019; Kazemi, 2018), habilitando a Computação em Cluster. A Computação em Cluster apresenta bom desempenho em ambiente de sistema distribuído, com características tais como: baixo consumo de energia, bom aproveitamento de armazenamento e boa taxa de utilização e adaptação do uso da rede de computadores e de comunicações. Bollier et al. (2010) destacam a capacidade da Computação em Cluster de fornecer um contexto favorável ao crescimento de dados. Já Miller et al. (2013) argumentam que a falta de disponibilidade de dados se torna cara, porque os usuários transferem mais decisões para métodos analíticos; o uso incorreto dos métodos e/ou pontos fracos inerentes podem produzir decisões erradas com alto custo.

O Hadoop é um framework de computação distribuída, desenvolvido em Java, voltado para clusters e processamento de grandes massas de dados. Após mapear os dados distribuídos e aplicar as transformações ou regras de negócio (processo MapReduce), o framework consolida o resultado e apresenta a resposta ao usuário final (Almeida et al., 2019; O’Driscoll et al., 2013; Kazemi, 2018). De acordo com Jadhav et al. (2018), a estrutura do Hadoop consiste em um sistema de arquivos distribuídos (Hadoop Distributed File System - HDFS) e outros projetos como Map Reduce, Yarn, Hive, Ping, Mahout, Avro, Sqoop, Oozie, Chukwa, Flume e Zookeeper chamados de ecossistema de componentes, que ajudam a resolver problemas de processamento de Big Data, como podemos observar na Figura 2.

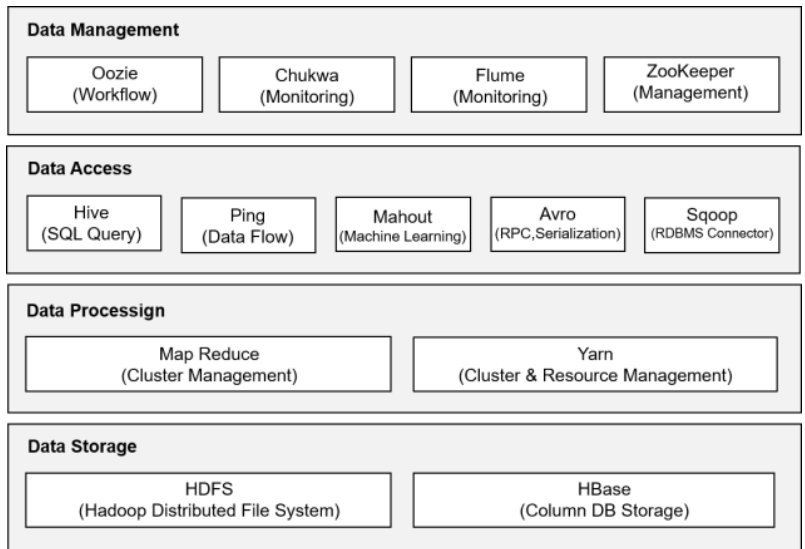

Figura 2. Jadhav et al. (2018) Componentes do ecossistema do Hadoop. Adaptado pelo autor

Estas relações entre os componentes do Ecossistema de Big Data, incluindo os processos e ferramentas para o trabalho com as massas de dados, são destacadas por Almeida et al. (2019). No referido trabalho, se verifica a relação com a Computação em Nuvem/distribuída, bem como com ferramentas e métodos de processamento de dados não utilizados na computação tradicional. Mais uma vez, até os sistemas de guarda de dados e a relação destes volumes com a era da informação e da demanda por alto poder computacional para decisões negociais são compreensíveis, além de ressaltar a complexidade do Ecossistema de Big Data.

Estas decisões e os processos para se obtê-las, por meio da análise de grandes volumes de dados, em uma análise preliminar, são a razão da existência do Big Data (Almeida et al., 2019). Razão esta que é reforçada no contexto da Administração Pública Federal, constituindo-se em ferramenta fundamental para apoiar os processos de tomada de decisões e de cumprimento dos normativos de transparência governamental. 


\subsection{Big Data no Setor Público}

Conforme citado na Seção 1, o setor público carece da utilização de ferramentas de Big Data, seja por questões de melhor controle das informações para tomada de decisões, seja pela necessidade de transparência das ações estatais junto à coletividade administrada.

Mesmo com estas características, o setor público encontra diferenças com relação a implantação do Big Data e seus ecossistemas. Segundo Klievink et al. (2017), o Big Data está sendo implementado com sucesso no setor privado e em pesquisas científicas. No entanto, o setor público parece estar ficando para trás. As organizações governamentais muitas vezes reconhecem as oportunidades relacionadas com Big Data, mas não se avaliam como aptas para a utilização de grandes volumes de dados, nem se consideram adequadamente equipadas para o Big Data. Os autores tratam exatamente acerca desta insegurança, apresentando uma estrutura para avaliar a prontidão das organizações públicas. O método utilizado busca desmistificar o conceito de Big Data para organizações do setor público holandês (Klievink et al. 2017), conforme expresso em termos de características organizacionais específicas e mensuráveis. Os resultados obtidos sugerem que as organizações podem ser tecnicamente capazes de utilizar as tecnologias de Big Data, mas não obterão ganhos significativos com essas atividades se os aplicativos não se ajustarem às suas organizações e às principais tarefas estatutárias.

De acordo com Janssen and Van Den Hoven (2015), considerando que além de serem massivos, os dados podem ser abertos, compondo o que é literariamente chamado de BOLD (do inglês, Big and Open Linked Data), a utilização de Big Data por entes governamentais resulta em novas oportunidades e tem o potencial de transformar o governo e suas interações com o público. Como efeito colateral, o BOLD oferece a oportunidade de analisar até mesmo o comportamento dos indivíduos, aumentar o controle e reduzir a privacidade. Ao mesmo tempo, em linha com as melhores práticas, o BOLD pode ser usado para criar um governo transparente, reforçando a abordagem dos autores mencionados anteriormente, e com os objetivos de implantação do Big Data em entes governamentais. Ainda segundo Janssen and Van Den Hoven (2015), transparência e privacidade são consideradas importantes valores sociais e democráticos, que estão mudando a forma como os cidadãos informados participam de processos democráticos. Os conceitos de transparência e privacidade têm muitas interpretações e são considerados difíceis de serem compreendidos, o que dificulta, por consequência, as formas de implementação dos sistemas relacionados com as informações detidas por entes estatais.

Considerando as premissas dos parágrafos anteriores, a motivação deste artigo é o estudo da implementação do Big Data no setor público brasileiro, buscando por trabalhos relacionados com os entes governamentais de outros países e brasileiros, com a premissa inicial de que as semelhanças entre os objetivos institucionais de órgãos governamentais guardam semelhanças embora em diferentes regiões. Passa-se, agora, à Seção 3 para entendimento dos procedimentos adotados.

\section{METODOLOGIA}

\subsection{Seleção dos Artigos}

O mapeamento sistemático está relacionado com o conhecimento da literatura correlata, que pode auxiliar no entendimento da questão em epígrafe. Desta forma, para atingir o objetivo proposto, o processo do Mapeamento Sistemático foi conduzido, conforme disposto na Figura 3. 


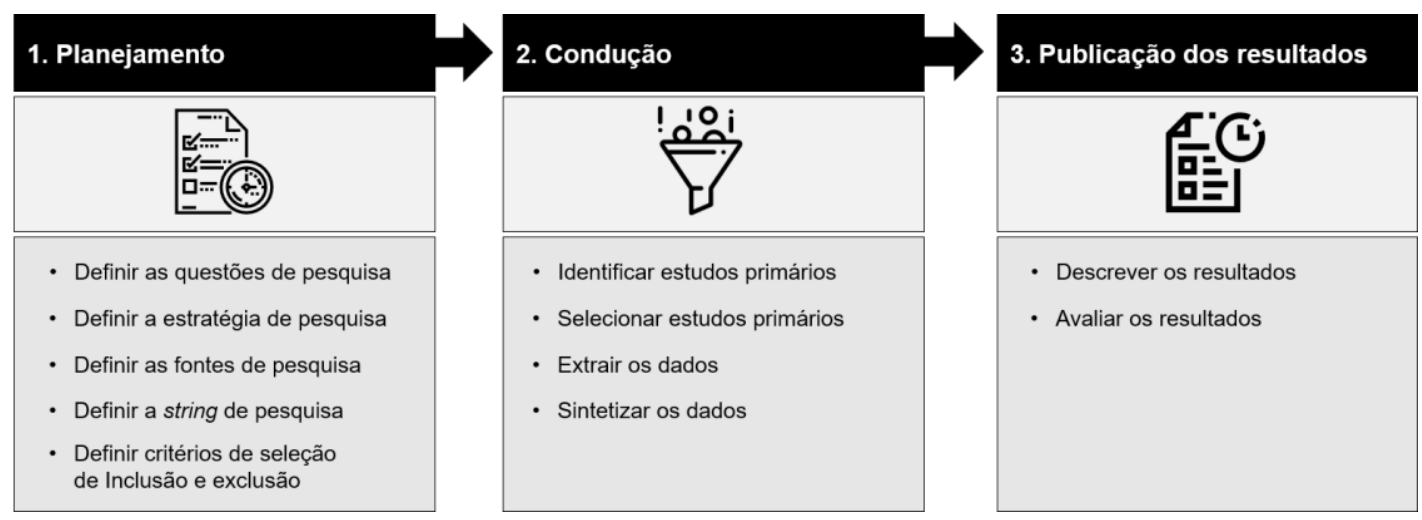

Figura 3. Condução do mapeamento sistemático. Autoria própria

Considerando os objetivos e os desafios da pesquisa citados anteriormente, chegamos à seguinte questão principal (QP):

(QP) Quais os objetivos e maiores desafios ou dificuldades de implantação de Big Data no setor público?

Complementarmente, como perguntas secundárias (PS), que, integradas, constroem o conhecimento necessário para responder à questão principal, listam-se:

(PS1) Quais os impactos econômicos nas soluções de Big Data no setor público?

(PS2) Quais as oportunidades e desafios da adoção de Big Data no setor público?

(PS3) Quais os impactos que práticas de Big Data trazem ao desenvolvimento de soluções?

Trata-se sobre mapeamento sistemático da literatura o processo pautado em:

1) Elaboração de "strings" de pesquisa, orientadas pela estratégia de busca;

2) Variedade de fontes de pesquisa para localização dos estudos;

3) Definição de critérios de inclusão e exclusão dos estudos selecionados;

4) Avaliação da qualidade metodológica das produções recuperadas.

5) Bases de dados consultadas e estratégias de busca.

O levantamento dos artigos foi realizado em três plataformas de pesquisa, conforme exposto na Tabela 1.

Tabela 1. Repositórios selecionados. Elaboração própria

\begin{tabular}{ll}
\hline Repositório & Endereço \\
\hline ACM Digital Library & $\mathrm{https} / / \mathrm{dl}$. acm.org/ \\
\hline IEEE Xplore & $\mathrm{https}: / /$ ieeexplore.iee.org/ \\
\hline ScienceDirect - Elsevie & $\mathrm{https} / / /$ www.sciencedirect.com/ \\
\hline
\end{tabular}

Considerando tais bases de pesquisa, algumas combinações de termos foram fundamentais para obtenção de artigos capazes de auxiliar no mapeamento sistemático e na obtenção do estado da arte. Procedeu-se ao cruzamento das principais palavras-chave relacionadas aos temas investigados, quais sejam: "Big Data", "Setor Público" e "Governo", além de outros eventualmente necessários para o enriquecimento das fontes de pesquisa, com objetivo de abarcar uma maior quantidade de produções, evitando que algum estudo muito importante ou de muito destaque fosse excluído no levantamento. Assim, algumas strings de pesquisa foram montadas e todas as publicações selecionadas foram referentes ao período de 2016 a 2020, conforme apresentadas na Tabela 2. Devido às particularidades dos motores de busca de cada plataforma utilizada, a string de busca principal precisou sofrer algumas adaptações, a fim de assegurar um resultado que englobasse um volume maior de estudos relevantes. 
Tabela 2. Strings de busca. Elaboração própria

\begin{tabular}{|c|c|c|}
\hline ID & Base de dados & Query utilizada \\
\hline 1 & ACM Digital Library & $\begin{array}{l}\text { "query": AllField: (“public sector") AND } \\
\text { AllField: ("Big Data") AND AllField: } \\
\text { ("difficulties" OR “opportunities" OR “economic } \\
\text { impacts" OR "economic advantages") "filter”: } \\
\text { Publication Date: (01/01/2016 TO 12/31/2020) }\end{array}$ \\
\hline 2 & IEEE Explore & $\begin{array}{l}\text { ("Big Data" AND "Government" OR "Big Data" } \\
\text { AND "Public Sector") }\end{array}$ \\
\hline 3 & ScienceDirect - Elsevier & $\begin{array}{l}\text { "Big Data" AND "Government" AND "Public } \\
\text { Sector" }\end{array}$ \\
\hline
\end{tabular}

Foram considerados alguns critérios de seleção para que um determinado estudo fosse incluído como passível de análise para a pesquisa, conforme apresentado na Tabela 3.

Tabela 3. Critérios de inclusão de artigos. Elaboração própria

\begin{tabular}{ll}
\hline CI & Critério de inclusão de artigos \\
\hline 1 & Apresenta os objetivos e maiores desafios/dificuldades de implantação de Big Data \\
\hline 2 & Aborda os impactos econômicos nas soluções de Big Data no Setor Público \\
\hline 3 & Aborda as oportunidades e desafios da adoção de Big Data \\
\hline
\end{tabular}

De maneira análoga, tomaram-se alguns critérios para exclusão de artigos que apareceram entre os resultados das queries apresentadas na Subseção 3.4, os quais estão descritos na Tabela 4.

Tabela 4. Critérios de exclusão de artigos. Elaboração própria

\begin{tabular}{ll}
\hline CE & Critério de exclusão de artigos \\
\hline 1 & Repetidos \\
\hline 2 & Muito semelhantes \\
\hline 3 & Não acessível \\
\hline 4 & Publicado como "artigo curto" ou somente "poster" \\
\hline 5 & Sem resumo ou abstract \\
\hline 6 & Publicado apenas resumo \\
\hline 8 & Versão mais antiga de outro artigo já considerado ou incluído \\
\hline
\end{tabular}

\subsection{Estudos dos Resultados}

Após a aplicação das strings de busca descritas na Tabela 2, em cada base dados, chegamos a um conjunto de estudos que estão sumarizados na Tabela 5. Para uma melhor visualização, os artigos foram agrupados por ano e por base de dados. Em seguida, as Tabelas 6 e 7 trazem o resultante de estudos após a aplicação dos critérios de inclusão e exclusão, respectivamente, também agrupados por base/ano.

Tabela 5. Artigos por base/ano. Elaboração própria

\begin{tabular}{lcccccc}
\hline Base de dados & 2016 & 2017 & 2018 & 2019 & 2020 & TOTAL \\
\hline ACM Digital Library & 35 & 41 & 55 & 53 & 17 & 201 \\
\hline IEEE Explore & 193 & 233 & 254 & 257 & 082 & 1019 \\
\hline Science Direct - Elsevier & 108 & 138 & 154 & 242 & 158 & 800 \\
\hline TOTAL GERAL & 336 & 412 & 463 & 552 & 257 & 2020 \\
\hline
\end{tabular}


Tabela 6. Artigos excluídos por base/ano. Elaboração própria

\begin{tabular}{lcccccc}
\hline Base de dados & 2016 & 2017 & 2018 & 2019 & 2020 & TOTAL \\
\hline ACM Digital Library & 35 & 40 & 51 & 51 & 17 & 194 \\
\hline IEEE Explore & 188 & 222 & 243 & 256 & 82 & 991 \\
\hline Science Direct - Elsevier & 106 & 135 & 152 & 241 & 154 & 788 \\
\hline TOTAL GERAL & 329 & 397 & 446 & 548 & 253 & 1973 \\
\hline
\end{tabular}

Tabela 7. Artigos selecionados por base/ano. Elaboração própria

\begin{tabular}{lcccccc}
\hline Base de dados & 2016 & 2017 & 2018 & 2019 & 2020 & TOTAL \\
\hline ACM Digital Library & 0 & 1 & 4 & 2 & 0 & 7 \\
\hline IEEE Explore & 5 & 11 & 11 & 1 & 0 & 28 \\
\hline Science Direct - Elsevier & 2 & 3 & 2 & 1 & 4 & 12 \\
\hline TOTAL GERAL & 7 & 15 & 17 & 4 & 4 & 47 \\
\hline
\end{tabular}

Para a priorização dos artigos, bem como das respostas das perguntas, foi aplicada a metodologia de enfoque meta-analítico, conforme descrito nos trabalhos de Mariano and Rocha (2017), Mariano et al. (2011) e Sánchez-Meca and López-Pina (2008). Essa abordagem tende a apresentar os trabalhos de maior relevância acadêmica ou com maior quantidade de citações acadêmicas em destaque dentro de um conjunto previamente obtido. Graficamente, as atividades de tal metodologia estão esquematizadas na Figura 4. Os detalhes de cada uma das três etapas representadas na aludida Figura 4 estão descritas em Mariano and Rocha (2017).

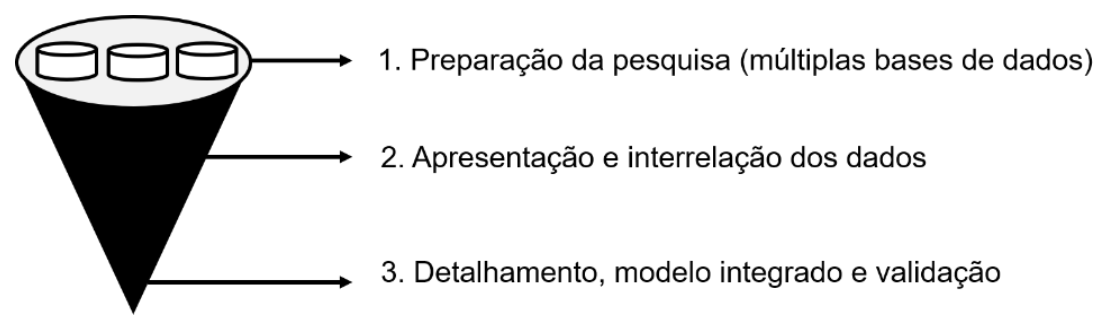

Figura 4. Diagrama Enfoque Meta Analítico. Extraído de Mariano and Rocha (2017)

No entanto, destaca-se que alguns artigos, não resultantes da priorização com o enfoque meta-analítico, bem como do procedimento principal descrito ao longo do presente trabalho, foram mantidos ou incluídos como base literária da pesquisa. Isso se deve ao fato de haver relação direta destes trabalhos com o objetivo desta pesquisa, como, por exemplo, o artigo de Victorino et al. (2017), que trata especificamente de Big Data no setor público brasileiro, embora ainda encontre poucas citações ou relevância dentro das bases pesquisadas.

Com fundamento nos estudos selecionados, algumas contribuições podem ser identificadas, possibilitando inferir respostas às perguntas secundárias PS1 e PS2 relatadas na Subseção 3.1. Tal resultado pode ser obtido por meio de enfoque em certos termos componentes da busca principal. O quantitativo de estudos selecionados, que apoiam as respostas às perguntas secundárias, está sintetizado na Tabela 8.

Tabela 8. Bases selecionadas. Elaboração própria

\begin{tabular}{lll}
\hline Base de Dados & PS1 & PS2 \\
\hline ACM Digital Library & 7 & 4 \\
\hline IEEE Xplore & 8 & 10 \\
\hline ScienceDirect - Elsevier & 4 & 5 \\
\hline
\end{tabular}

Graficamente, considerando os procedimentos descritos entre as Subseções 3.1 a 3.5, sintetiza-se os resultados conforme apresentados na Figura 5. 


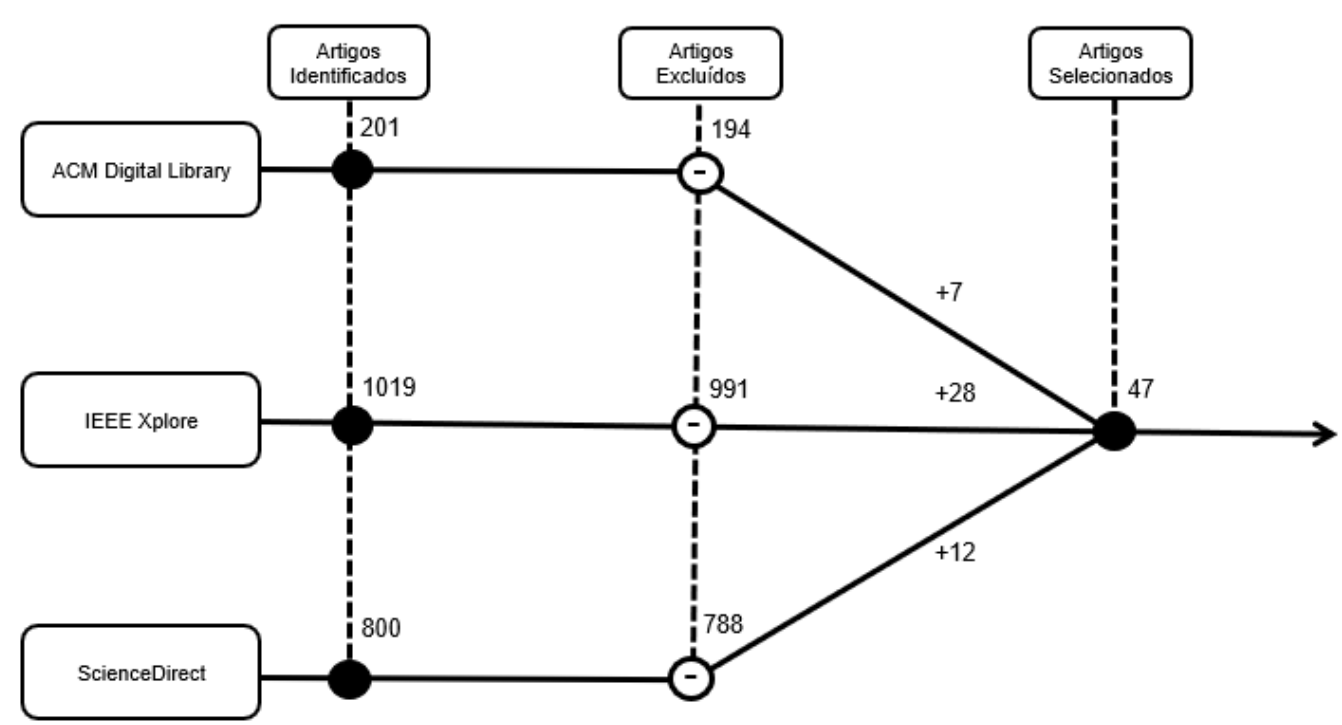

Figura 5. Síntese dos resultados dos procedimentos da pesquisa bibliográfica. Elaboração própria

Passa-se agora para a Seção 4, na qual se apresentam as discussões.

\section{DISCUSSÕES}

Para selecionar artigos capazes de auxiliar na resposta da PS1 (impactos econômicos), pode-se focar nos artigos que citam impactos econômicos e criação de valor para organizações, por meio da adoção de Big Data, no caso, no setor público. Com este diapasão, estão sendo identificadas contribuições como as de Khtira et al. (2017) ou mesmo de Klievink et al. (2017), as quais mencionam as possibilidades de criação de valor em diferentes áreas de atuação dos órgãos governamentais, cada qual em seu segmento. Igualmente, citam uma melhor eficiência quanto à análise e coleta de informações e na interação com a comunidade administrada, permitindo, em última análise, melhor emprego dos recursos públicos ou mesmo sua economia.

Para a PS2 (oportunidades e desafios), foram encontradas contribuições no artigo de Klievink et al. (2017), o qual relata entre as dificuldades de implementação do Big Data no setor público, inicialmente, a dúvida sobre a capacidade, incluindo aspectos de infraestrutura computacional e da adoção de ferramentas de Big Data. Em adição, é informado no aludido trabalho que possuir a infraestrutura e as ferramentas pouco têm a acrescentar se o trabalho de Big Data não estiver muito correlacionado com a missão institucional de um determinado órgão público ou governamental. Ou seja, não acrescenta valor se não houver um projeto de Big Data conectado à missão institucional.

Ainda referente à PS2, Khtira et al. (2017) citam, ainda, que o Big Data no setor público se torna uma necessidade também em virtude das novas maneiras de a sociedade interagir com os órgãos governamentais. Essa participação popular é capaz de gerar uma enorme quantidade de dados, as quais as organizações públicas podem e devem saber manusear e tratar de forma a obter informação capaz de conduzir políticas públicas e orientar a tomada de decisão. As oportunidades de aplicações do Big Data para o setor público vão desde grandes aplicações em bases de dados de governos nacionais, passando pela aplicação de serviços públicos em comunidades locais, como exemplificado pelo trabalho de Malomo and Sena (2017).

Finalmente, considerando a PS3 (impactos), as dificuldades na implementação do Big Data estão muito ligadas aos desafios citados na PS2, em especial quando se consideram as organizações públicas. Inicialmente, o já citado artigo de Klievink et al. (2017) aponta a questão da compreensão da capacidade de implementação, considerando aspectos de infraestrutura computacional. Igualmente, a dificuldade de construir um projeto de Big Data realmente capaz de agregar valor significativo à missão institucional do órgão em questão. $\mathrm{O}$ artigo de Malomo and Sena (2017) também enfatiza que as organizações públicas, neste caso representadas por seu corpo técnico-funcional, muitas vezes não dominam as questões sobre como o Big Data pode ser útil no crescimento de eficiência das atividades públicas. Os artigos não se furtam, porém, a citar também dificuldades 
técnicas, relacionadas, por exemplo, com a multiplicidade de fontes de dados, muitas vezes derivadas de dados não estruturados, principalmente em virtude da forma como são coletados.

A pesquisa, conforme mencionado em epígrafe, encontra considerável relevância, uma vez que as dificuldades de implementação do Big Data podem ser comuns a diversas entidades públicas e até mesmo privadas. As eventuais soluções, por sua vez, em virtude de muitas dificuldades serem comuns, podem se constituir em soluções que auxiliem, portanto, diversas organizações.

Tem-se, portanto, que a principal questão a ser tratada no trabalho ora proposto é: Quais os objetivos e maiores desafios e dificuldades de implantação do Big Data no setor público? Segundo Victorino et al. (2017), os objetivos das organizações governamentais, ao participar de um ambiente de Big Data, estão diretamente relacionados com as suas funções de fornecimento de serviços públicos e com a necessidade de provimento de informações aos cidadãos, incluindo questões relacionadas com a transparência de governo, o que corrobora, mesmo que este autor tenha escrito seus trabalhos com vistas ao caso brasileiro, o explicitado pelos citados artigos utilizados na análise das questões descritas.

Na revisão foram encontrados desafios para a implantação de Big Data no setor público (Al-Sai and Abualigah, 2017). Como forma de responder à questão principal deste trabalho, os objetivos e desafios estão classificados em perspectiva, conforme Tabela 9.

Tabela 9. Desafios e possíveis soluções propostas. Adaptado de Al-Sai and Abualigah (2017)

\begin{tabular}{ll}
\hline Desafios & Soluções Possíveis \\
\hline Perspectiva de & $\bullet$ Aumentar a capacidade e utilização do armazenamento disponível, como recursos em \\
Tecnologia & nuvem para armazenamento e processamento de Big Data. \\
& - Implementar questões de segurança questões de política, legislação e regulamentação. \\
& Usar ferramentas eficazes de gerenciamento de dados para analisar dados de estrutura, \\
& desestruturação e estrutura simulada de várias fontes. \\
& - Fornecer e integrar recursos eficientes de Big Data
\end{tabular}

\begin{tabular}{|c|c|}
\hline $\begin{array}{l}\text { Perspectiva de } \\
\text { Pessoas }\end{array}$ & $\begin{array}{l}\text { - Aprimorar as habilidades do capital humano disponível em Big Data, como análise de } \\
\text { dados e segurança de dados; e importar especialistas que podem apoiar a implementação de } \\
\text { Big Data. } \\
\text { - Implementar uma iniciativa nacional que apoia a colaboração entre Big Data e governo } \\
\text { eletrônico. } \\
\text { - Aumentar a conscientização sobre Big Data e o impacto no governo eletrônico. } \\
\text { - Permitir que os cidadãos sejam mais criativos e se expressem efetivamente nas redes } \\
\text { sociais. }\end{array}$ \\
\hline $\begin{array}{l}\text { Perspectiva de } \\
\text { Processos de } \\
\text { Negócio }\end{array}$ & $\begin{array}{l}\text { - Desenhar uma estratégia eficaz que suporte o roteiro para a implementação de Big Data no } \\
\text { governo eletrônico. } \\
\text { - Estabelecer parcerias públicas e privadas. } \\
\text { - Criar uma rede entre comunidade e governo sob a égide de Big Data. } \\
\text { - Formular estratégias eficazes e um roteiro que leve a um ambiente de Big Data. } \\
\text { - A liderança deve motivar, envolver, influenciar o suporte à implementação de Big Data no } \\
\text { governo eletrônico. } \\
\text { - Integrar um grande volume de dados valiosos no governo eletrônico aos processos de } \\
\text { - Comada de decisão. } \\
\text { estratégico e decisões. }\end{array}$ \\
\hline
\end{tabular}

Além dos desafios supracitados, existem ainda outros obstáculos para o tema virar realidade dentro do setor público. Estudos de outros países, como no caso da Turquia, demonstram que o custo, infraestrutura insuficiente, questões de privacidade e segurança, burocracia governamental, falta de legislação sobre o tema, adaptação dos servidores ao processo e a integração dos sistemas de TI existentes são grandes obstáculos para a efetiva adoção de soluções de Big Data (Dogdu et al., 2018). 


\section{CONCLUSÃO}

Este artigo apresenta um mapeamento sistemático sobre o tema de implantação do Big Data no setor público, como pesquisa componente de esforços na verificação de como e porque os órgãos do Governo Brasileiro implantam as aludidas soluções de Big Data.

A consulta de publicações recentes sobre o tema tem fornecido subsídios para formulação das respostas sobre as perguntas de pesquisa, bem como sobre ferramentas e processos para adoção de tais soluções, as quais podem maximizar a produtividade no desenvolvimento de software e implantação de Big Data.

É importante destacar que foram pesquisados e analisados principalmente estudos internacionais, de acordo com a proposta inicial de bases adotadas, assim como dos critérios, tomando como premissa que o caso brasileiro guarda semelhanças com os casos internacionais já documentados. A semelhança é sugerida nas poucas publicações brasileiras sobre o tema.

As dificuldades levantadas em diversas organizações públicas, ainda que não brasileiras, podem ser comuns a diversos tipos de organização que pretendam implantar Ecossistemas de Big Data no Brasil, tanto públicas, nas diversas esferas de governo, quanto privadas.

A revisão sistemática permitiu que se formulassem direcionadores das futuras pesquisas sobre implantação de Big Data nas organizações públicas brasileiras, organizados pelas perspectivas de tecnologia, de pessoas e de processos de negócio, abordando questões de infraestrutura e conhecimento tecnológico, objetivos estratégicos das organizações públicas e suas missões institucionais, bem como sua forte relação com questões de publicidade e transparência e da eficiência do gasto público.

Devido ao baixo número de artigos publicados sobre o contexto brasileiro, espera-se que este estudo venha a atenuar esse cenário, orientando estudos futuros, que poderão focar em documentar a realidade brasileira e descrever casos junto às organizações governamentais (nos diferentes poderes e nas diferentes esferas governamentais), levantando particularidades relevantes e intrínsecas ao contexto público brasileiro, algo que não foi possível identificar com o mapeamento sistemático reportado neste estudo.

\section{AGRADECIMENTOS}

Os autores agradecem o apoio das Agências Brasileiras de Pesquisa, Desenvolvimento e Inovação CAPES (concessão 23038.007604/2014-69 FORTE), CNPq (concessão 465741/2014-2 INCT em Segurança Cibernética) e a FAP-DF (concessões 0193.001366/2016 UIoT e 0193.001365/2016 SSDDC), bem como as cooperações com o Ministério da Economia (TED DIPLA 005/2016), Advocacia Geral da União (TED AGU 697.935/2019) e a CESAR School.

\section{REFERÊNCIAS}

Akoka, J., Comyn-Wattiau, I. and Laoufi, N., 2017. Research on Big Data-A systematic mapping study. Computer Standards \& Interfaces, 54, 105-115. [Online]. Disponível em: https://linkinghub.elsevier.com/retrieve/pii /S0920548917300211.

Al-Sai, Z. A. and Abualigah, L. M., 2017. Big data and E-government: A review. In 2017 8th International Conference on Information Technology (ICIT), pp. 580-587, IEEE. doi: 10.1109/ICITECH.2017.8080062.

Almeida, W. H. C., de Aguiar Monteiro, L., de Lima, A. C., Hazin, R. R. and Escobar, F., 2019. Survey on Trends in Big Data: Data Management, Integration and Cloud Computing Environment. SOFTENG 2019: The Fifth International Conference on Advances and Trends in Software Engineering, p. 7.

Araújo Júnior, R. H. and de Sousa, R. T. B., 2016. Estudo do ecossistema de Big Data para conciliação das demandas de acesso, por meio da representação e organização da informação. Ciência da Informação, 45(3). [Online]. Disponível em: http://www.periodicos.ufpb.br/index.php/pbcib/article/view/39276.

Barbieri, C., 2001. BI-business intelligence: Modelagem e Tecnologia. Axcel Books, 2001. [On-line]. Disponível em: https://books.google.com.br/books?id=HD1KAAAACAAJ.

Bollier, D., Firestone, C. M. and others, 2010. The promise and peril of big data. Aspen Institute, Communications and Society Program Washington, DC.

Davenport, T., 2014. Big data at work: dispelling the myths, uncovering the opportunities. Harvard Business Review Press. 
Dean, J. and Ghemawat, S., 2008. MapReduce: simplified data processing on large clusters. Communications of the ACM, 51(1), 107-113. [Online]. Disponível em: http://portal.acm.org/citation.cfm?doid=1327452.1327492.

Demchenko, Y., De Laat, C. and Membrey, P., 2014. Defining architecture components of the Big Data Ecosystem. In 2014 International Conference on Collaboration Technologies and Systems (CTS) (pp. 104-112). IEEE. [Online]. Disponível em: http://ieeexplore.ieee.org/document/6867550/.

Dogdu, E., Ozbayoglu, M., Yazici, A. and Karakaya, Z., 2018. Perceptions, Expectations and Implementations of Big Data in Public Sector, 2018 3Rd Int. Conf. Comput. Sci. Eng., pp. 615-620.

Schimdt, E., 2010, "Every 2 days we create as much information as we did up to 2003," [Online]. Disponível em: http://techcrunch.com/2010/08/04/schmidt-data/.

Kim, G. H., Trimi, S. and Chung, J. H., 2014. Big-data applications in the government sector, Communications of the ACM, vol. 57, no. 3, pp. 78-85. [Online]. Disponível em: http://dl.acm.org/citation.cfm?doid=2566590.2500873.

Gantz, J. and Reinsel, D., 2011. Extracting value from chaos. IDC iview, 1142(2011), 1-12.

Miller, H. E. and others, 2013. Big-data in cloud computing: A taxonomy of risks, Information Research, vol. 18, no. 1, pp. $18-1$

Hashem, I. A. T., Yaqoob, I., Anuar, N. B., Mokhtar, S., Gani, A. and Khan, S. U., 2015. The rise of "big data" on cloud computing: Review and open research issues. Information Systems, 47, 98-115. [Online]. Disponível em: https://linkinghub.elsevier.com/retrieve/pii/S0306437914001288.

Inmon, W. H. and Linstedt, D., 2014. Data architecture: a primer for the data scientist: big data, data warehouse and data vault. Morgan Kaufmann.

Jadhav, B., Patankar, A. B. and Jadhav, S. B., 2018. A Practical approach for integrating Big data Analytics into E-governance using hadoop. 2018 Second International Conference on Inventive Communication and Computational Technologies (ICICCT). doi:10.1109/icicct.2018.8473353.

Janssen, M. and Van Den Hoven, J., 2015. Big and Open Linked Data (BOLD) in government: A challenge to transparency and privacy? [Online]. Disponível em: https://linkinghub.elsevier.com/retrieve/pii/S0740624X15001069

Kazemi, U., 2018. A Survey of Big Data: Challenges and Specifications. CiiT International Journal of Software Engineering and Technology, Vol 10, No 5.

Klievink, B., Romijn, B. J., Cunningham, S. and de Bruijn, H., 2017. Big data in the public sector: Uncertainties and readiness. Information systems frontiers, 19(2), 267-283. [Online]. Disponível em: http://link.springer.com/10.1007/s10796-016-9686-2.

Laney, D., 2001. Application delivery strategies. META Group, Stamford.

Malomo, F. and Sena, V., 2017. Data intelligence for local government? Assessing the benefits and barriers to use of in the public sector. Policy \& Internet, 9(1), 7-27. [Online]. Disponível em: http://doi.wiley.com/10.1002/poi3.141

Mariano, A. M. and Rocha, M. S., 2017. Revisão da literatura: apresentação de uma abordagem integradora. In AEDEM International Conference (Vol. 18).

Mariano, A. M., García Cruz, R. and Arenas Gaitán, J., 2011. Meta análises como instrumento de pesquisa: Uma revisão sistemática da bibliografia aplicada ao estudo das alianças estratégicas internacionais. Congresso Internacional de Administração [Online]. Disponível em: http://rgdoi.net/10.13140/RG.2.1.1150.1522.

Márquez, F. P. G. and Lev, B., 2017. Big data management. Springer.

O’Driscoll, A., Daugelaite, J. and Sleator, R. D., 2013. 'Big data', Hadoop and cloud computing in genomics. Journal of biomedical informatics, 46(5), 774-781.

Khtira, R., Elasri, B. and Rhanoui, M., 2017. From Data to Big Data: Moroccan Public Sector, in Proceedings of the 2nd International Conference on Cloud and Applications - BDCA'17. Tetouan, Morocco: ACM Press, pp. 1-6. [Online]. Disponível em: http://dl.acm.org/citation.cfm?doid=3090354.3090401.

Ramos, A., 2015. Infraestrutura Big Data Com Opensource. Ciencia Moderna. [On-line]. Available: https://books.google.com.br/books?id=Y7HjswEACAAJ.

Sánchez-Meca, J. and López-Pina, J. A., 2008. El enfoque meta-analítico de generalización de la fiabilidad. [The Meta-analytic approach of reliability generalization], Acción Psicológica, vol. 5, no. 2, pp. 37-64. [Online]. Disponível em: http://revistas.uned.es/index.php/accionpsicologica/article/view/457.

Shin, D. H. and Choi, M. J., 2015. Ecological views of big data: Perspectives and issues. Telematics and Informatics, 32(2), 311-320. [Online]. Disponível em: https://linkinghub.elsevier.com/retrieve/pii/S0736585314000665.

Victorino, M. C., Shiessl, M., Oliveira, E. C., Ishikawa, E., de Holanda, M. T. and de Lima Hokama, M., 2017. Uma proposta de ecossistema de big data para a análise de dados abertos governamentais concetados. Informação \& sociedade, 27(1)., p. 19. 